\title{
Cosmic-Ray Acceleration in Centaurus A
}

\author{
Frank M. Rieger \\ Max-Planck-Institut für Kernphysik, 69117 Heidelberg, Germany \\ E-mail: frank.rieger@mpi-hd.mpg.de
}

The apparent clustering of ultra-high energy cosmic-ray (UHECR) events along Cen A has attracted considerable attention within recent years. We analyze the potential of classical acceleration mechanisms to produce UHECR particles under conditions expected for Cen A. Mechanisms discussed include DC electric field acceleration in the vicinity of the black hole, first-order Fermi acceleration at internal shocks in the jet and stochastic particle acceleration in the giant radio lobes. The results indicate that: (1) Efficient acceleration of protons to $E_{c}=5 \times 10^{19} \mathrm{eV}$ and beyond remains challenging and most likely requires the operation of an additional mechanism capable of boosting energetic seed protons up by a factor of $\sim 10$. It is proposed that shear acceleration along the large-scale jet in Cen A could be a promising candidate for this. (2) Heavier elements, such as iron nuclei, are more easily accelerated (by, e.g., shocks or direct electrostatic fields) and may not need additional boosting to reach $E \geq E_{c}$. Observations may therefore reflect the contributions of more than one mechanism. (3) If Cen A indeed proves to be an UHECR emitting source, one may expect the cosmic-ray composition to become heavier above energies of a few times $10^{19} \mathrm{eV}$.

25th Texas Symposium on Relativistic Astrophysics

December 6-10, 2010

Heidelberg, Germany 


\section{Introduction}

The apparent clustering of ultra-high-energy cosmic-ray (UHECR) events along the radio galaxy Centaurus A (Cen A), as indicated by the Pierre Auger Observatory (PAO) analysis, has renewed the interest into nearby AGN as efficient UHECR accelerators.

Based on data up to August 2007, the PAO Collaboration initially reported evidence for an anisotropy at the $99 \%$ confidence level in the arrival directions of cosmic rays with energies above $\sim 6 \times 10^{19}$ $\mathrm{eV}$ [1]. The anisotropy was measured by the fraction of arrival directions that were less than $\sim 3^{\circ}$ from the positions of nearby AGN (within $75 \mathrm{Mpc}$ ) from the VCV catalog. While that correlation has now decreased using the newly available (twice as large) data set, the updated analysis still suggests that a region of the sky around the position of Cen A has the largest excess of arrival directions relative to isotropic expectations [2]. This obviously motivates a theoretical investigation of possible UHECR acceleration sites in Cen A. Below we analyze the efficiency constraints expected for a number of acceleration mechanisms when applied to Cen A. As it turns out, most mechanisms have serious difficulties in accelerating protons beyond a few times $10^{19} \mathrm{eV}$. While the experimental situation is not fully conclusive yet, this result may fit into recent PAO indications for an increase of the average mass composition with rising energies up to $E \simeq 10^{19.6} \mathrm{eV}$ [3].

\section{The radio galaxy Cen $A$}

Cen $\mathrm{A}$ is the nearest active galaxy, only a distance of $d \sim 3.4 \mathrm{Mpc}$ away. Being a spectacular example of a gas-rich disk galaxy consumed in a merger with a giant elliptical galaxy, it displays a peculiar radio morphology with two jets emerging from its nucleus, and giant radio lobes stretching out to $250 \mathrm{kpc}$ and extending over a 8 x 4 degree field on the sky, e.g. see [4] for a review. VLBI observations suggest that Cen A is a non-blazar source with its inner jet inclined at a rather large viewing angle $i \gtrsim 50^{\circ}$ and characterized by relatively modest bulk flow speeds $u_{j} \sim 0.5 \mathrm{c}$ $[5,6]$. The center of its activity is a supermassive black hole with mass $m_{\mathrm{BH}}=(0.5-3) \times 10^{8} M_{\odot}$ $[8,9]$. Given its bolometric luminosity output of the order of $L_{b} \sim 10^{43} \mathrm{erg} / \mathrm{s}$ [10], Cen A is rather under-luminous and believed to accrete at sub-Eddington rates. If the inner disk in Cen A remains cooling-dominated (standard disk), accreting rates $\dot{m} \sim 10^{-3} \dot{m}_{\text {Edd }}$ and equipartition magnetic field strengths close to the black hole of order $B_{0} \sim\left(2 L_{b} / r_{g}^{2} c\right)^{1 / 2} \sim 2 \times 10^{3} \mathrm{G}$ might be expected (where $r_{g}=G M / c^{2} \simeq 1.5 \times 10^{13} \mathrm{~cm}$ is the gravitational radius for a $10^{8} M_{\odot}$ black hole). If the disk switches to a radiatively inefficient (RIAF) mode, characteristic magnetic field strengths may be somewhat higher, possibly reaching $B_{0} \sim 10^{4} \mathrm{G}$.

\section{Particle acceleration in Cen A}

The above noted source characteristics can be used to evaluate the efficiency of possible acceleration mechanisms:

\subsection{Particle acceleration in the vicinity of the black hole}

Rotating magnetic fields, either driven by the disk or the black hole itself, can facilitate the acceleration of charged particles emerging from the vicinity of the black hole: 
- Direct electrostatic field acceleration: If the black hole is embedded in a poloidal field of strength $B_{p}$ and rotating with angular frequency $\Omega_{H}$, it will induce an electric field of magnitude $|\vec{E}| \sim\left(\Omega_{H} r_{H}\right) B_{p} / c$. This corresponds to a voltage drop across the horizon $r_{H}$ of magnitude $\Phi \sim r_{H}|\vec{E}|$. In terms of the electric circuit analogy, a rotating black hole thus behaves like a unipolar inductor (battery) with non-zero resistance, so that power can be extracted by electric currents flowing between its equator and poles. For Cen A, the voltage drop is expected to be of order

$$
\Phi \sim 3 \times 10^{19} a\left(\frac{m_{\mathrm{BH}}}{10^{8} M_{\odot}}\right)\left(\frac{B_{p}}{10^{4} \mathrm{G}}\right)[\mathrm{V}],
$$

where $0 \leq a \leq 1$ denotes the dimensionless Kerr parameter. If a charged particle (with charge number $Z$ ) can fully tap this potential, particle acceleration to ultra-high energies

$$
E=Z e \Phi \sim 3 \times 10^{19} \mathrm{ZeV}
$$

may become possible. This would then suggest a rather heavy composition instead of a light one (e.g., iron nuclei instead of protons) for cosmic ray events above $E_{c}=5 \times 10^{19} \mathrm{eV}$.

Yet, whether such energies can, in fact, be achieved, seems questionable: (1) First, the charge density produced by annihilation of $\mathrm{MeV}$ photons emitted by an RIAF in Cen A most likely exceeds the Goldreich-Julian (GJ) density required to screen the electric field [11]. If so, then a non-negligible part of the field will not be available for particle acceleration. (2) Secondly, even if no significant screening would occur, curvature losses [12] would constrain achievable proton energies to values of

$$
E_{p} \lesssim 10^{19} a^{1 / 4}\left(M / 10^{8} M_{\odot}\right)^{1 / 2}\left(B_{p} / 10^{4} \mathrm{G}\right)^{3 / 4} \mathrm{eV} .
$$

(3) Thirdly, large-scale poloidal fields with strengths of $B_{p} \sim 10^{4} \mathrm{G}$ would be required. This seems overly optimistic, at least in the case of a standard disk [13]. (4) Fourthly, a highly spinning black hole with $a \sim 1$ would be required although rather modest spins may be expected for FR I sources [14]. Taken these four points together, efficient DC acceleration of protons to energies of $E_{c}$ and beyond in Cen A seems unlikely, while it could be (marginally) possible for heavier elements.

- Centrifugal particle acceleration: Even if the charge density would exceed the GJ density, particle acceleration due to inertial effects (i.e., centrifugal acceleration along rotating magnetic fields) could still be possible, e.g. see $[15,16]$. If one requires that the associated acceleration timescale remains larger than the inverse of the relativistic gyro-frequency [16], the maximum Lorentz factor for cosmic ray acceleration in Cen A becomes

$$
\gamma_{\curvearrowright} \times 10^{7} \gamma_{0}^{1 / 3} Z^{2 / 3}\left(\frac{m_{p}}{m_{0}}\right)^{2 / 3}\left(\frac{r_{\mathrm{L}}}{10^{14} \mathrm{~cm}}\right)^{2 / 3}
$$

where $r_{\mathrm{L}}$ denotes the light cylinder radius (typically of a few $r_{g}$ ). This suggests that centrifugal acceleration in Cen A will be unable to account for the production of UHECR. 


\section{Fermi-type particle acceleration in the jets and beyond}

Suppose that particle acceleration is Fermi-type, i.e., due to multiple scattering off moving magnetic inhomogeneities, with a small energy change in each scattering event. The characteristic energy gain per scattering event for an energetic charged particle (velocity $v \sim c$ ), elastically scattering off some magnetic irregularity moving with typical speed $\vec{u}$, is given by

$$
\Delta \varepsilon:=\varepsilon_{2}-\varepsilon_{1}=2 \Gamma^{2}\left(\varepsilon_{1} u^{2} / c^{2}-\vec{p}_{1} \cdot \vec{u}\right),
$$

where $\Gamma=\left(1-u^{2} / c^{2}\right)^{-1 / 2}$ is the Lorentz factor of the scatterer, $\vec{p}=\varepsilon \vec{v} / c^{2}$ the particle momentum and the indices 1 and 2 denote particle properties before and after scattering. A particle can thus gain or lose energy depending on whether it suffers head-on/approaching $\left(\vec{p}_{1} \cdot \vec{u}<1\right)$ or following/overtaking $\left(\vec{p}_{1} \cdot \vec{u}>1\right)$ collisions. We may then distinguish the following scenarios (e.g., $[17,18,19])$ :

- Diffusive shock (1st order Fermi) acceleration: In the case of diffusive shock acceleration, energetic charged particles are assumed to pass unaffected through the shock front and, by being elastically scattered in the fluid on either side, to cross and re-cross the shock several times. Sampling the difference $\Delta u$ in flow velocities across a shock (always head-on), the characteristic energy gain for a particle crossing the shock, cf. eq. (4.1), becomes first order in $\Delta u / c$, i.e., $\Delta \varepsilon / \varepsilon_{1} \propto(\Delta u / c)$. As this energy gain is acquired during a shock crossing time $t_{c} \sim \lambda / u_{s}$ (with $u_{s}$ the shock speed and $\lambda$ the scattering mean free path), the characteristic acceleration timescale (for a non-relativistic shock) is of the order of

$$
t_{\mathrm{acc}} \sim \frac{\varepsilon}{(d \varepsilon / d t)} \sim\left(\frac{\varepsilon_{1}}{\Delta \varepsilon}\right) t_{c} \sim \lambda \frac{c}{u_{s}^{2}} .
$$

If radiative losses are negligible, we can equate this timescale with the one for cross-field diffusion out of the system, $t_{e} \sim r_{w}^{2} /(\lambda c)$, or the dynamical timescale, $t_{d} \sim z / u_{s}$ (whichever is smaller), to derive an estimate for the maximum achievable particle energy, cf. [20]

$$
E_{\max } \sim Z e B r_{w} \beta_{s} \sim 2 \times 10^{19} Z\left(\frac{B_{0}}{10^{4} \mathrm{G}}\right)\left(\frac{\beta_{s}}{0.1}\right) \mathrm{eV},
$$

taking $\lambda \sim r_{\text {gyro }}$ to be of the order of the gyro-radius, $\beta_{s}=u_{s} / c$, and $B(z) \sim 4 B_{0}\left(r_{g} / z \alpha_{j}\right)$ for the typical magnetic field strength at location $z$ (allowing for magnetic field compression by up to a factor of 4 ). $B_{0}$ is the field strength close to the black hole, and $\alpha_{j}$ is the jet opening angle. Radio observations indicate only mildly relativistic jet speeds $u_{j} \sim 0.5$ c. If representative for the general flow, then typical internal shock speeds should be rather moderate with $\beta_{s} \sim 0.1$ or less. This seems supported by the nuclear SED of Cen A, showing a synchrotron peaks below $10^{20} \mathrm{~Hz}$ (already assuming that the 2 nd peak is not due to inverse Compton, cf. [21, 22]): Synchrotron-limited electron shock acceleration gives a (magnetic field-independent) peak at $\sim 3 \times 10^{19}\left(\beta_{s} / 0.1\right)^{2} \mathrm{~Hz}$, consistent with rather modest shock speeds. Equation (4.3) suggests that if shock acceleration would be responsible for UHECR production in Cen A, then the expected composition should be rather heavy, i.e., efficient shock acceleration of protons to energies of $\sim E_{c}$ and beyond seems unlikely. 
Moreover, it can be shown that acceleration of protons beyond a few times $10^{19} \mathrm{eV}$ would require a jet power well in excess of the one expected for Cen A as an FR I source (e.g., [23]).

- Stochastic 2nd order Fermi acceleration: Equation (4.1) implies that the average energy gain due to scattering off randomly moving magnetic inhomogeneities is only second order, i.e., $\Delta \varepsilon / \varepsilon_{1} \propto(u / c)^{2}$. Thus, in order to achieve efficient acceleration, the velocity $u$ of the scatterers has to be sufficiently large. As the energy gain is acquired over a scattering time $t_{s} \sim \lambda / c$, the characteristic acceleration timescale is given by

$$
t_{\mathrm{acc}} \sim \frac{\varepsilon}{(d \varepsilon / d t)} \sim\left(\frac{c}{v_{A}}\right)^{2} \frac{\lambda}{c},
$$

where we have assumed that scattering is due to Alfvén waves moving with speed $u=v_{A}=$ $B / \sqrt{4 \pi \rho}$. If we again neglect radiative losses, achievable particle energies are limited by escape via cross-field diffusion, resulting in an upper limit of

$$
E_{\max } \sim 2 \times 10^{19} Z\left(\frac{R}{100 \mathrm{kpc}}\right)\left(\frac{v_{A}}{0.1 c}\right)\left(\frac{B}{10^{-6} \mathrm{G}}\right) \mathrm{eV},
$$

on scales of $R \sim 100 \mathrm{kpc}$, as appropriate for the giant radio lobes in Cen A. If relativistic Alfvén speeds $\left(v_{A} \gtrsim 0.3 \mathrm{c}\right.$ ) would occur, then 2 nd order Fermi effects could possibly allow acceleration of protons to ultra-high energies [24]. Yet, most likely the conditions are less favorable (see also [25]): A thermal origin of the observed X-ray emission in the giant lobes of Cen A, as suggested in [26], for example, would imply thermal plasma densities $n_{\text {th }} \sim\left(10^{-5}-10^{-4}\right) \mathrm{cm}^{-3}$. The resultant Alfvén speeds $v_{A} \sim 0.003 \mathrm{c}$ would then simply be too small. Such high values for the thermal plasma density are in fact consistent with independent estimates based on Faraday rotation measurements in the radio lobes of Cen A [27]. It seems thus less likely, that efficient UHECR acceleration could take place in the giant radio lobes of Cen $\mathrm{A}$.

- Shear acceleration: Suppose that the background flow, in which the scatterers are thought to be embedded, has a smoothly (gradually) changing velocity profile in the direction perpendicular to the jet axis (e.g., a shear flow or layer with $\vec{u}=u_{z}(r) \vec{e}_{z}$ ). Once energetic particles are scattered across the flow, they may well be able to sample the flow difference $d u$ and thereby get accelerated [28, 29]. Like stochastic 2nd order Fermi acceleration, the average energy gain in this process is proportional to $(d u / c)^{2}$, although the physical origin is clearly different (i.e., due to the systematic, instead of the random motion of the scatterers). The velocity difference in the flow, that a particles experiences by being scattered across, is $d u \sim\left(d u_{z} / d r\right) \lambda$, where $\lambda$ is the scattering mean free path of the particle. Again, this energy change is acquired over a mean scattering time $\tau_{s} \sim \lambda / c$, so that the characteristic acceleration timescale becomes

$$
t_{\mathrm{acc}} \sim \frac{\varepsilon_{1}}{\Delta \varepsilon / \tau_{s}} \sim \frac{1}{\left(d u_{z}(r) / d r\right)^{2}} \frac{c}{\lambda} .
$$

Compared to eq. (4.2) and eq. (4.4), the acceleration timescale is now inversely proportional to $\lambda$. Thus, as a particle increases its energy (so that its mean free path $\lambda \propto p^{\alpha}, \alpha>0$, 
becomes larger), the acceleration timescale decreases. Shear acceleration will, therefore, preferentially pick up high energy seed particles for further energization, and act more easily on protons than on heavier nuclei. In general, it seems well possible that shocks, operating in the jet (either on smaller scales or within a spine), could provide the energetic seed protons required for further shear acceleration along the jet [30]. If so, then maximum achievable particle energies are expected to be essentially determined by the confinement condition (i.e., gyro-radius smaller than width of the shear layer). The large-scale jet in Cen A has a projected length of about $4.5 \mathrm{kpc}$, and towards its end a width of about $\sim 1 \mathrm{kpc}$ [7,31]. If we take a characteristic magnetic field strength of $B \sim 10^{-4} b_{j} \mathrm{G}$ on kpc-scale (cf. the magnetic flux conservation argument) and assume that the width of the shear becomes comparable to the width of the jet, then achievable maximum energies would be of the order of

$$
E \sim \mathrm{ZeB}(\Delta r) \sim 10^{20} b_{j} \mathrm{ZeV}
$$

This suggests that shear acceleration might be able to boost energetic seed protons (e.g., produced by shock acceleration) up to energies beyond $E_{c}$. Note that in the presence of sufficient internal shear, magnetic field amplification by stretching and folding of field lines could occur $[32,33]$, in which case we may well have $b_{j} \gtrsim 1$. A shear dynamo effect could possibly also explain why in Cen A the magnetic field direction appears to be almost parallel along the kpc jet [6].

\section{Conclusions}

From a theoretical point of view, efficient acceleration of protons to UHECR energies in Cen A remains challenging for most standard mechanisms. Observational evidence for UHE protons may therefore support the operation of an additional acceleration mechanism such as shear. As indicated above, this could allow to boost achievable particle energies beyond $E_{c}=5 \times 10^{19} \mathrm{eV}$. Efficient shear acceleration in Cen A would require high energy seed particles which, however, could be provided by, e.g., shock acceleration. A fraction of these seed protons may then be picked up and accelerated to the maximum energy given by the confinement limit. If such a two-step process would indeed take place, spectral changes in the cosmic ray energy spectrum may not only be due to propagation effects. The situation is much more relaxed for heavier elements like iron nuclei. Such particles are most likely directly accelerated (either by shocks or within the black hole magnetosphere) to energies of $E_{c}$ and beyond. If Cen A would indeed be an efficient UHECR accelerator one may thus expect the composition to become heavier above energies $\sim 10^{19} \mathrm{eV}$.

\section{References}

[1] The Pierre Auger Collaboration, Science 318 (2007) 938.

[2] The Pierre Auger Collaboration, Astroparticle Physics 34 (2010) 314.

[3] The Pierre Auger Collaboration, Phys. Rev. Lett. 104 (2010) 091101.

[4] F.P. Israel, A\&ARv 8 (1998) 237. 
[5] S. Tingay et al., AJ 115 (1998) 960.

[6] M.J. Hardcastle et al., ApJ 593 (2003) 169.

[7] J.O. Burns, E.D. Feigelson, and E.J. Schreier, ApJ 273 (1983) 128.

[8] A. Marconi et al., A\&A 448 (2006) 921.

[9] N. Neumayer et al., ApJ 671 (2007) 1329.

[10] D. Whysong and R. Antonucci, ApJ 602 (2004) 116.

[11] A. Levinson \& F.M. Rieger, ApJ (2010) in press.

[12] A. Levinson, Phys. Rev. Lett. 85 (2000) 912.

[13] M. Livio, G.I. Ogilvie, and J.E. Pringle, ApJ 512 (1999) 100.

[14] R. Daly, ApJ 691 (2009) L72.

[15] Z. Osmanov, A. Rogava, and G. Bodo, A\&A 470 (2007) 395.

[16] F.M. Rieger and F.A. Aharonian, IJMPD 17 (2008) 1569.

[17] J.G. Kirk and R.O. Dendy, JPhG 27 (2001) 1589.

[18] P. Duffy and K.M. Blundell, PPCF 47 (2006) 667.

[19] F.M. Rieger, V. Bosch-Ramon, and P. Duffy, Ap\&SS 309 (2007) 119.

[20] A.M. Hillas, ARA\&A 22 (1984) 425.

[21] J.M. Bai and M.G. Lee, ApJ 549 (2001) L173.

[22] J.-P. Lenain et al., A\&A 478 (2008) 111.

[23] F.M. Rieger, eprint arXiv:0911.4004

[24] M.J. Hardcastle et al., MNRAS 393 (2009) 1041.

[25] S. O'Sullivan, B. Reville, and A.M. Taylor, MNRAS 400 (2009) 248 .

[26] N. Isobe et al., ASP Conf. Ser. 250 (2001) 394.

[27] I.J. Feain et al., ApJ 707 (2009) 114.

[28] J.R. Jokipii and G.E. Morfill, ApJ 356 (1990) 255.

[29] F.M. Rieger and P. Duffy, ApJ 652 (2006) 1044.

[30] F.M. Rieger and F.A. Aharonian, A\&A 506 (2009) L41.

[31] R.P. Kraft et al., ApJ 569 (2002) 54.

[32] M.C. Begelman, R.D. Blandford and M.J. Rees, Rev. Mod. Phys. 56 (1984) 255.

[33] V. Urpin, A\&A 455 (2006) 779. 